\title{
An All-Solid Dispersion-Compensating Photonic Crystal Fiber Based on Mode Coupling Mechanism in Dual-Concentric Core
}

\author{
Zhaolun Liu $\mathbb{D}^{1,2}$ Chunlan Zhang, ${ }^{1}$ and Yuwei $Q \mathbf{u}^{2,3}$ \\ ${ }^{1}$ School of Information Science and Engineering, Yanshan University, Qinhuangdao 066004, China \\ ${ }^{2}$ The Key Laboratory for Special Optical Fiber and Fiber Sensor of Hebei Province, Yanshan University, \\ Qinhuangdao 066004, China \\ ${ }^{3}$ State Key Laboratory of Information Photonics and Optical Communications, \\ Beijing University of Posts and Telecommunications, Beijing 100876, China
}

Correspondence should be addressed to Zhaolun Liu; liuzhaolun@ysu.edu.cn

Received 7 July 2020; Revised 7 October 2020; Accepted 24 November 2020; Published 9 December 2020

Academic Editor: Paramasivam Senthilkumaran

Copyright (c) 2020 Zhaolun Liu et al. This is an open access article distributed under the Creative Commons Attribution License, which permits unrestricted use, distribution, and reproduction in any medium, provided the original work is properly cited.

An all-solid dispersion-compensating photonic crystal fiber based on mode coupling mechanism in dual-concentric core has been proposed. The mode coupling characteristics, dispersion, confinement loss of the fiber, and the influence on dispersion of some structure parameters are simulated by full-vector finite element method. By using the relationship between phase matching wavelengths and coupling strength with the change of fiber microstructure parameters, an all-solid dual-concentric-core dispersion-compensating photonic crystal fiber is presented. The structure parameters on dispersion characteristic are investigated. The results demonstrate that the proposed fiber has a large negative dispersion value $8465 \mathrm{ps} /(\mathrm{nm} \cdot \mathrm{km})$ at $1550 \mathrm{~nm}$. The effective mode area and the splicing loss to the standard single mode fiber are $12.8 \mu \mathrm{m}^{2}$ and $1.89 \mathrm{~dB}$ at $1550 \mathrm{~nm}$, respectively. At $1550 \mathrm{~nm}$, the confinement loss is less than $1 \times 10^{-3} \mathrm{~dB} / \mathrm{km}$ and the bending loss with $2 \mathrm{~cm}$ bending diameter is less than $1 \times 10^{-2} \mathrm{~dB} / \mathrm{km}$.

\section{Introduction}

In modern high-speed optical fiber communication system, the cumulative dispersion in transmission process is the main factor limiting the communication rate [1]. Therefore, the dispersion compensation for high-speed optical fiber communication system has become a hot research topic. Photonic crystal fiber (PCF) cladding contains a lot of micro air holes with periodic or disordered distribution. Because of this special structure, this kind of fiber has a high degree of design freedom, and it can flexibly adjust the dispersion characteristics of fiber [2]. It allows to arbitrarily shape the dispersion in a wide spectral range [3].

Birks et al. got the dispersion compensation photonic crystal fiber (DCPCF) for the negative dispersion value as $-2000 \mathrm{ps} /(\mathrm{nm} \cdot \mathrm{km})$ at $1550 \mathrm{~nm}$ by using control effect of large air hole and small fiber core on light to increase the influence of waveguide dispersion [4]. Gerome et al. proposed the structure of dual-concentric-core dispersion- compensating photonic crystal fiber (DCC-DCPCF) [5] and used the mode coupling mechanism of dual-concentric-core (DCC) to obtain the large negative dispersion. After then, the design and realization of dispersion compensation characteristics by using microstructural fiber are mostly based on the mode coupling mechanism of DCC [6-9]; the transmission mode between the inner and outer core is regulated to achieve the conversion of light energy between the inner and outer core, so as to flexibly adjust the dispersion compensation characteristics of the optical fiber. All the fibers mentioned above are air holes or liquid-filled air holes. A liquid-filled hybrid structure of DCC-DCPCF with the negative dispersion value being $-40400 \mathrm{ps} /(\mathrm{nm} \cdot \mathrm{km})$ at $1550 \mathrm{~nm}$ and an inner core high-doping DCC-DCPCF with the negative dispersion value being $-51625 \mathrm{ps} /(\mathrm{nm} \cdot \mathrm{km})$ at $1550 \mathrm{~nm}$ were reported by $\mathrm{Hsu}$ et al. [10, 11]. Partha et al. designed a DCC-DCPCF with liquid-filled air holes-the negative dispersion value has reached $-52100 \mathrm{ps} /(\mathrm{nm} \cdot \mathrm{km})$ at $1550 \mathrm{~nm}$ - and analyzed the influence of the material on 
the phase matching wavelength $(\lambda p)$ and dispersion value at different temperatures [12]. Han et al. developed a negative dispersion value of $-41800 \mathrm{ps} /(\mathrm{nm} \cdot \mathrm{km})$ at $1550 \mathrm{~nm}$ in a DCC-DCPCF by using two methods to expand the first-layer air hole [13]. DCC-DCPCF introduced above are air hole silica or liquid-filled air holes structure, which have disadvantages of complex drawing technique and not easy to be coupled with standard single-mode fiber (SSMF).

Liquid-filled air holes fiber belongs to the fiber postprocessing technology; it is very difficult to achieve liquid filling due to the DCC-DCPCF has a small structure, and this kind of fiber is also not conducive to the application of practical engineering. An all-solid PCF with low dispersion slope and an all-solid PCF with a wide wavelength bands' dispersion compensation were reported by Xu et al. [14, 15]. Mariusz et al. used an all-solid PCF with flattened normal dispersion to improve spectral flatness of supercontinuum [16]. Wang et al. developed an all-solid dual-concentric-core microstructure fiber for ultra-broadband dispersion compensation, the designed fiber has very wide compensation bandwidth of up to $190 \mathrm{~nm}$. [17]. The all-solid PCF consists of low Ge-doped inner core and F-doped concentric ring inner cladding.

In this paper, based on the mode coupling mechanism between the traditional air hole silica DCC-DCPCF, the mode coupling characteristics of the DCC all-solid photonic crystal fiber are studied by the full-vector finite element method. The influence of the different structure parameters on the mode coupling characteristics is analyzed. The structure parameters on dispersion characteristic are investigated. Based on that, we optimize the fiber structure parameters reasonably and design an all-solid DCC-DCPCF. In addition, the all-solid structure provides a more friendly process for both fabricating and splicing; the designed allsolid DCC-DCPCF is easily fabricated and can easily access the existing optical network systems.

\section{Fundamental Theory}

A full-vector finite element method with the perfectly matched layer boundary conditions is used to analyze the mode coupling mechanism of all-solid DCC-DCPCF [18].

Starting with Maxwell's curl equations, the vector equation for the electric field vector $E$ can be derived as

$$
\left(\nabla_{i}^{2}+k_{0}^{2} n_{i}^{2}-\beta^{2}\right) E=0
$$

where the subscript $i$ is section number, $k_{0}=2 \pi / \lambda$ is the wave number in the vacuum, and $\lambda$ is operation wavelength.

The curvilinear hybrid edge/nodal elements based on linear tangential and quadratic normal vector basis functions are adopted to realize the computational window divisions and perfectly matched layer is incorporated as the boundary condition to absorb waves out of the computational window. Applying the finite element procedure to equation (1), the following eigenvalue equation is defined as

$$
[A]\{H\}=n_{\mathrm{eff}}^{2}[B]\{H\},
$$

where $[A]$ and $[B]$ are the global finite element matrices. The eigenvector $\{H\}$ and the eigenvalue $n_{\text {eff }}^{2}$ provide, respectively, the full vector magnetic field distribution on the cross section of PCF and the effective index of the mode.

The total dispersion $D(\lambda)$ of the fiber consists of two parts: the material dispersion $D_{\mathrm{m}}(\lambda)$ and the waveguide dispersion $D_{\mathrm{w}}(\lambda)$; that is [19],

$$
D(\lambda)=D_{\mathrm{w}}(\lambda)+D_{\mathrm{m}}(\lambda)
$$

The waveguide dispersion can be expressed as [20]

$$
D_{w}(\lambda)=-\frac{\lambda}{c} \frac{d^{2} \operatorname{Re}\left[n_{\mathrm{eff}}\right]}{d \lambda^{2}},
$$

where $c$ is the velocity of light in vacuum, $\lambda$ is the wavelength, and $\operatorname{Re}\left[n_{\text {eff }}\right]$ is the real part of the effective refractive index of the fundamental mode.

The material dispersion is defined as [21]

$$
D_{m}(\lambda)=-\frac{\lambda}{c} \frac{d^{2} n_{m}}{d \lambda^{2}}
$$

where $n_{\mathrm{m}}$ is the refractive index of the material, and it is obtained by Sellmeier's equation at different wavelengths.

The effective mode field area of the fiber can be expressed as [22]

$$
A_{\mathrm{eff}}=\frac{\left(\iint_{s}|E|^{2} d x d y\right)^{2}}{\iint_{s}|E|^{4} d x d y,}
$$

where $E$ is an electric field vector and $S$ represents the mode field area of the fiber.

The curved PCF can be replaced by a straight fiber with equivalent refractive index as [23]

$$
n(x, y)=n_{0}(x, y)\left(1+\frac{x}{R}\right)
$$

where $R$ is the curvature diameter and $n_{0}(x, y)$ is the original refractive index profile of the PCF.

The confinement loss is obtained from the imaginary part of $n_{\text {eff }}$ by

$$
L=\frac{20}{\ln 10} \frac{2 \pi}{\lambda} \operatorname{Im}\left[n_{\mathrm{eff}}\right] \times 10^{9} .
$$

The splice loss between the proposed DCC-DCPCF and the SSMF is theoretically evaluated according to the following definition [24]:

$$
L_{\text {splice }}=-20 \log \left[\frac{2 \omega_{1} \omega_{2}}{\omega_{1}^{2}+\omega_{2}^{2}}\right] \text {, }
$$

where $\omega_{1}$ and $\omega_{2}$ represent the mode field diameter of the DCC-DCPCF and SSMF.

\section{Structural Design and Simulation Analysis}

3.1. All-Solid DCC-DCPCF. The transverse section and the equivalent refractive index of the all-solid DCC-DCPCF are shown in Figure 1. The base material is pure silica, and 
the refractive index $n_{0}=1.45$. The fiber consists of a highgermanium-doped silica column as an inner core and a low-doped fluoride or boron silica column as the outer core or cladding and the refractive index of the doped rod is controlled within \pm 0.05 . It can be seen that the cladding structure contains three kinds of different size and different refractive index of medium cylinders, whose center medium cylinder is the inner core; its refractive index is $n_{1}$ and diameter is $d_{1}$; the outer core is the minimum media cylinder; its refraction index and diameter is $n_{2}$ and $d_{2}$, respectively; gray medium column is cladding and the diameter is $d_{3}$. The cladding refraction index contents $n_{3}<n_{2}<n_{0}<n_{1}$. The column spacing between any two silica columns is $\Lambda$, the number of low-doped silica columns between the inner core and outer core is $N$, and the number of layers after the outer core is $N_{\mathrm{o}}$. The silica columns of PCF have a hexagonal distribution. The first ring low-doped silica columns has 6 columns, the second ring low-doped silica columns has 12 columns, and the $N$ th ring low-doped silica columns has $6 * N$ columns. As for outer core, the low-doped silica columns number of the $N_{\mathrm{o}}$ th is $6 *\left(N_{\mathrm{o}}+N\right)$. For every two adjacent layers in outer cladding, there are six more columns in the outer layer than in the inner layer. There are no air holes in allsolid DCC-DCPCF, which make it easier to fabricate such fiber than conventional air-hole PCF. As long as the diameter of columns is not low than $0.10 \mu \mathrm{m}$, it can be fabricated.

3.2. Mode Coupling Characteristics of the All-Solid DCC$D C P C F$. To study the mode coupling characteristics of the all-solid DCC-DCPCF, a set of initial parameters is given: $n_{1}=1.480, n_{2}=1.445, n_{3}=1.42, d_{1}=1.30 \mu \mathrm{m}, d_{2}=0.51 \mu \mathrm{m}$, $d_{3}=2.40 \mu \mathrm{m} \Lambda=2.90 \mu \mathrm{m}, N=2$, and $N_{\mathrm{o}}=2$. The relationship between the wavelength and the effective refractive index of the inner core fundamental mode and outscore secondorder mode is calculated by the full-vector finite element method.

When the optical fibers are designed to have only inner core or outer core, the relationship between the wavelength and the effective refractive index of the inner core or outer core fundamental mode is shown in Figure 2. We can see that the effective refractive index of the inner core and outer core fundamental mode is approximately linear with the wavelength when it only has inner core or outer core; the effective refractive index of the inner core fundamental mode is greater than the outer core at the short wave, while it is the opposite at the long wave, the two effective refractive index curves have intersection points at a fixed wavelength. But if the structure of designed fiber is DCC, the effective refractive index of the inner core and outer core mode is abrupt in a certain wavelength, and the distribution of the mode field is also changed, which have the two modes of the outer core and inner core distribution, respectively.

According to the coupled mode theory, the core mode of DCC fiber affects each other by evanescent wave. The DCC fiber forms a super mode with guide characteristics by controlling of mode coupling mechanism between the inner

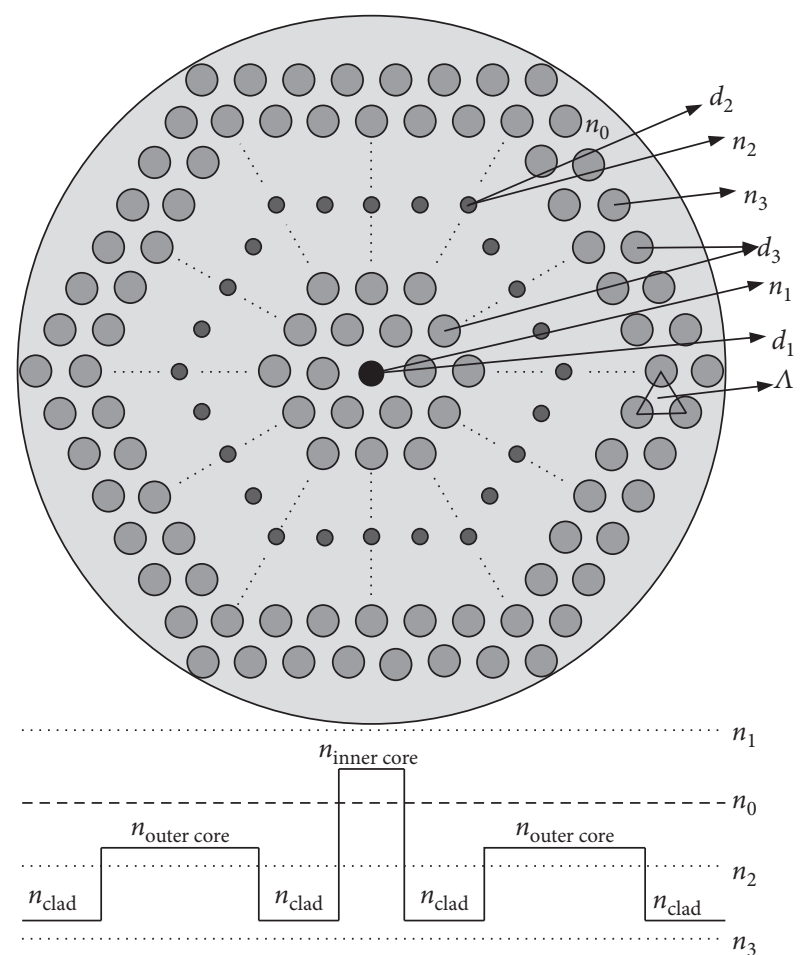

Figure 1: The transverse section and equivalent refractive index distribution for studied all-solid DCC-DCPCF.

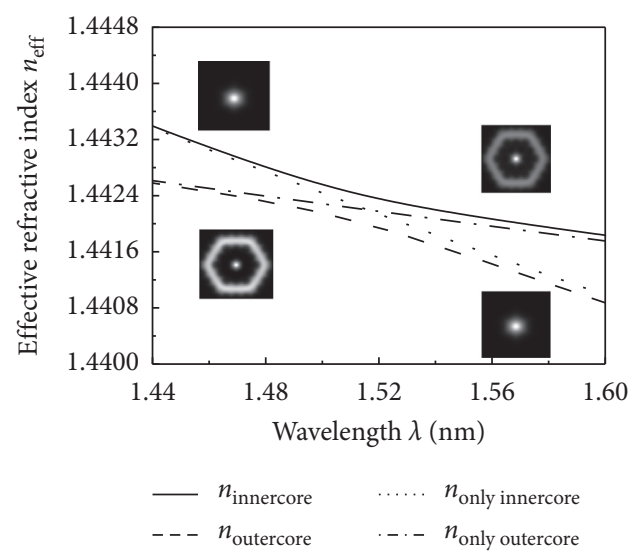

FIgURE 2: Effective index of inner core fundamental (solid line), outer core second-order mode (dashed line), and only inner core or only outer core fundamental mode (dotted or dash-dotted line). Insets show the modal field distributions of inner core fundamental mode and outer core second-order mode.

core and outer core. The inner core mode and the outer core mode in Figure 2 are called fundamental supermodel and second-order supermodel, respectively. At the short wavelength, the fundamental supermodel is transmitted in the inner core, and the second-order supermodel is transmitted in the outer core. Moreover, most of the energy of the fundamental supermodel and the second-order supermodel is gathered in the inner core and the outer core, respectively. With the increase of wavelength, the effective refractive index of the two modes has a sudden change in a certain 
wavelength. This wavelength is called $\lambda_{\mathrm{p}}$. At the $\lambda_{\mathrm{p}}$, the energy of the fundamental supermodel is gradually converted to the outer core, while the energy distribution of the second-order supermodel converts to the inner core gradually. After $\lambda_{\mathrm{p}}$, the energy of the fundamental supermodel is more and more transferred from the inner core to the outer core, and the energy of the second-order supermodel is more and more transferred from the outer core to the inner core.

The no-cross effect in the coupling process makes the effective refractive index curve of the fundamental supermodel and the second-order supermodel rapidly change, and the two modes produce large negative dispersion and positive dispersion, respectively, at $\lambda_{\mathrm{p}}$, as shown in Figure 3. Owing to the fact that SSMF has certain positive dispersion in common communication band, the large negative dispersion produced by the fundamental supermodel can compensate the cumulative dispersion in the transmission process. Due to the large loss of the second-order supermodel, it will decay continuously during transmission, so we do not need to consider the influence of second-order supermodel on communication.

3.3. Simulation Analysis. According to the refractive index distribution of the fiber transverse section, a change on the diameter of the medium cylinder is the equivalent to a change on the refractive index of the medium cylinder. The influence of the diameter of the medium cylinder on mode coupling characteristics is analyzed. When the diameter of inner core medium cylinder is changed, the relationship between the effective refractive index of the fundamental supermodel and the wavelength is shown in Figure 4. We can see when $d_{1}$ is changed, the effective refractive index of the fundamental supermodel at long wavelength is almost unchanged; the effective refractive index at short wavelength increases with the increase of $d_{1}$. At $d_{1}=0.90 \mu \mathrm{m}$, the effective refractive index of the fundamental supermodel has a distinct change-point, which is the phase matching point. With the increase of $d_{1}$, the abrupt change of the effective refractive index of the fundamental supermodel gradually becomes slow, but the mutation position can still be seen.

Shown in the insets of Figure 4(a), the fundamental supermodel is transmitted in the inner core at the short wavelength; most of the energy of the fundamental supermodel is gathered in the inner core for $d_{1}=0.90 \mu \mathrm{m}$. At the long wavelength, the fundamental supermodel is transmitted in the outer core; most of the energy of the fundamental supermodel is gathered in the outer core; that is, most of the energy is transferred from the inner core to the outer core. With $d_{1}=1.70 \mu \mathrm{m}$, most of the energy of the fundamental supermodel is also gathered in the inner core at the short wavelength, but at the long wavelength, the fundamental supermodel still has a large part of the energy gathered in the inner core, which means the energy transfer from the inner core to the outer core is slow. Thus, when the effective refractive index of the fundamental supermodel changes drastically from short wavelength to long wavelength, there is an obvious phase matching point. The energy of the fundamental supermodel is transferred rapidly from

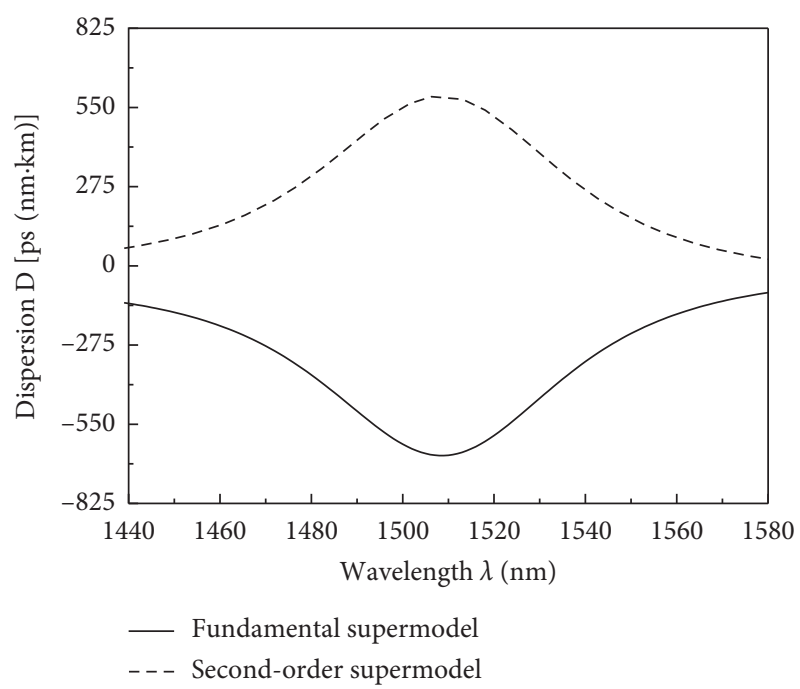

FIGURE 3: Large negative dispersion generated (solid line) by the fundamental supermodel and large positive dispersion (dashed line) generated by the second-order supermodel.

the inner core to the outer core near the phase matching point; that is, the supermodel between the inner core and outer core has a strongly coupling. When the effective refractive index of the fundamental supermodel changes slowly from short wavelength to long wavelength, there is no obvious phase matching point. The energy of the fundamental supermodel is transferred slowly from the inner core to the outer core near the phase matching point that means the supermodel between the inner core and outer core has a weak coupling. When the equivalent refractive index difference between the inner core and outer core increases, the effective index difference of the fundamental supermodel in the inner core and outer core is also increased. When the effective refractive index difference is small, the coupling process of the fundamental supermodel from inner core to outer core is fast and the coupling strength is large, producing a larger negative dispersion value. When the effective refractive index difference is large, the coupling process is slow and the coupling strength is weak, so a smaller negative dispersion value is generated. Hence, the coupling strength between the inner core and outer core can be expressed by the absolute value of the negative dispersion value, and the greater the absolute value is, the greater the coupling strength is. The variation of $\lambda_{\mathrm{p}}$ and coupling strength with $d_{1}$ is shown in Figure $4(\mathrm{~b}) . \lambda_{\mathrm{p}}$ produces red shift and the coupling strength of the fundamental supermodel decreases gradually with the increasing of $d_{1}$, which is consistent with the theoretical analysis.

Variation of the effective refractive index of the fundamental supermodel with wavelength when $d_{2}$ is changed can be seen in Figure 5(a). The effect of $d_{2}$ change and $d_{1}$ change is exactly the opposite. The effective refractive index of the fundamental supermodel at the short wavelength is basically unchanged, but the effective refractive index at the long wavelength is increased with the decrease of $d_{2}$. In fact, the equivalent refractive index of outer core becomes larger and the equivalent refractive index difference between inner 


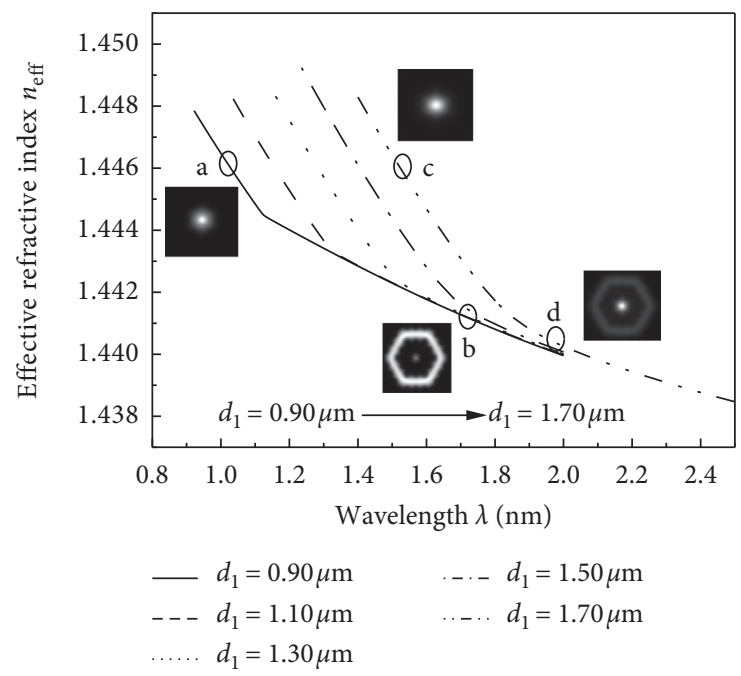

(a)

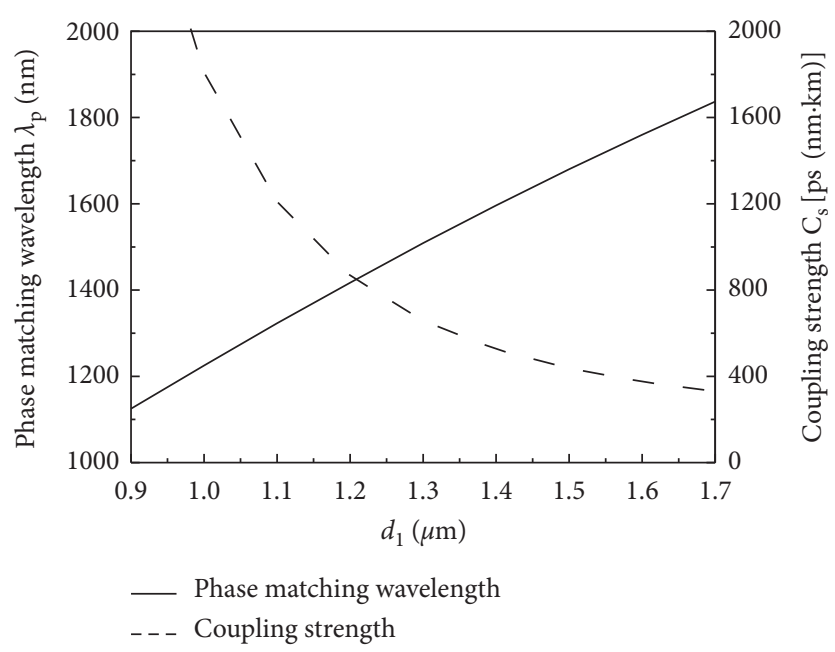

(b)

FIGURE 4: Variation of the effective refractive index of the fundamental supermodel with wavelength when $\mathrm{d} 1$ is changed (a) and $\lambda p$ and the coupling strength with $\mathrm{d}_{1}$ (b). Insets show the modal field distributions of fundamental supermodel at different wavelengths for $d_{1}=0.90 \mu \mathrm{m}$ and $d_{1}=1.70 \mu \mathrm{m}$.

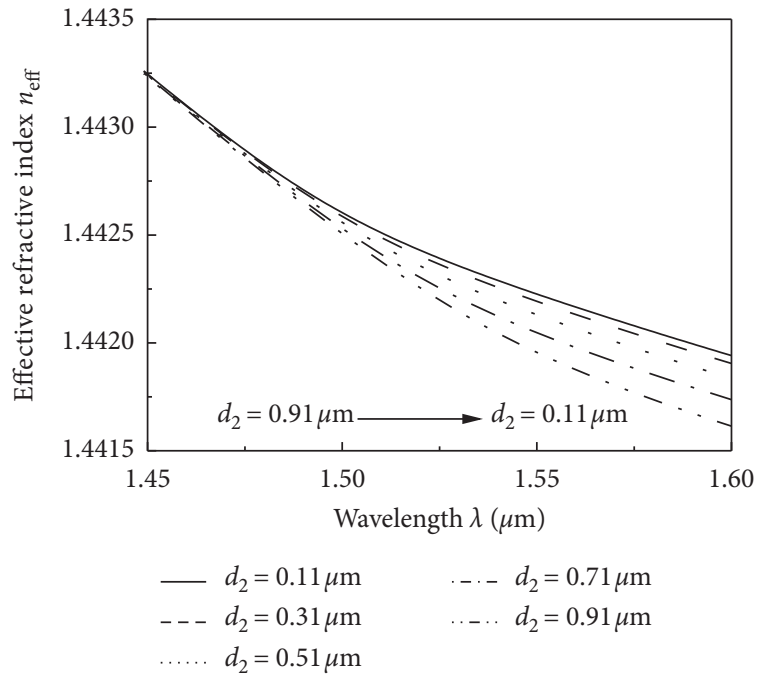

(a)

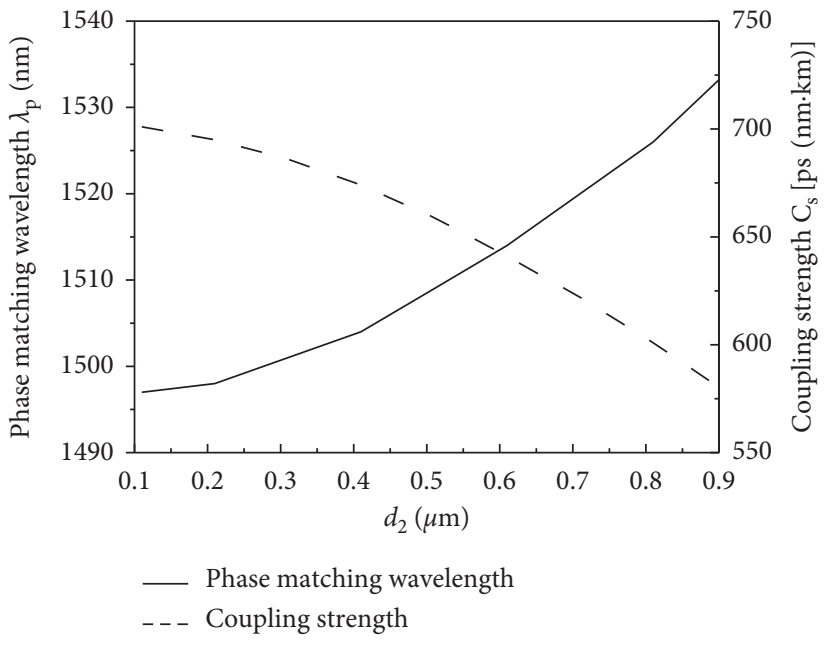

(b)

Figure 5: Variation of the effective refractive index of the fundamental supermodel with wavelength when $d_{2}$ is changed (a) and $\lambda p$ and the coupling strength with $d_{2}$ (b).

core and outer core decreases with the increase of $d_{2}$. It is equivalent to the equivalent refractive index difference of the fundamental supermodel transferred from the inner core to the outer core becomes smaller, which leads to a rapid coupling process of the fundamental supermodel from the inner core to the outer core and a larger coupling strength. The variation of $\lambda_{\mathrm{p}}$ and coupling strength with $d_{2}$ is presented in Figure 5(b). Figure 5(b) shows that $\lambda_{\mathrm{p}}$ produces red shift and the coupling strength of the fundamental supermodel decreases little by little with the increasing of $d_{2}$.
Variation of the effective refractive index of the fundamental supermodel with wavelength when $d_{3}$ is changed is analyzed in Figure 6(a). Decreasing $d_{3}$ increases the equivalent refractive index of the cladding, which is equal to the increase of the effective refractive index of the fundamental supermodel at the short wavelength and the long wavelength with the decrease of $d_{3}$, but the increase at the short wavelength is less than that at the long wavelength. This result is different from that which only changes the core area effective refractive index of designed fiber. At the same 


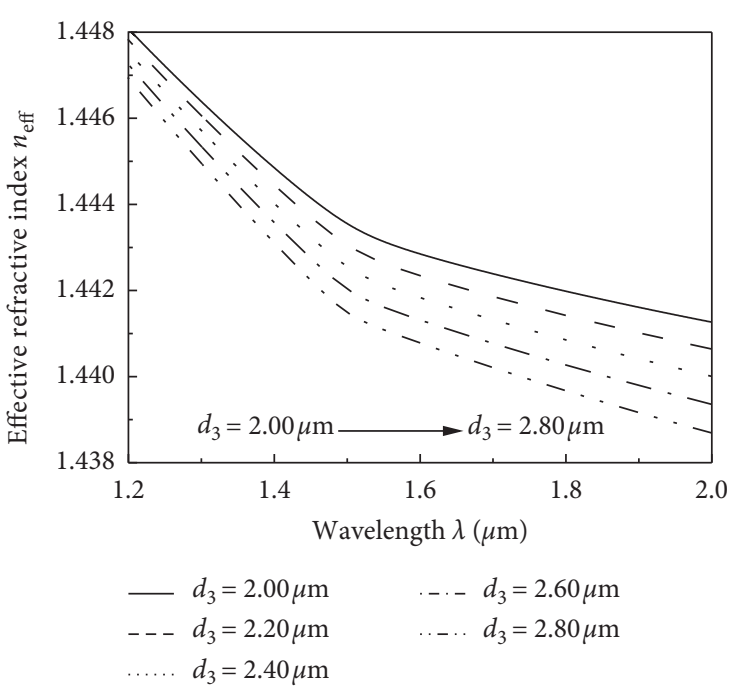

(a)

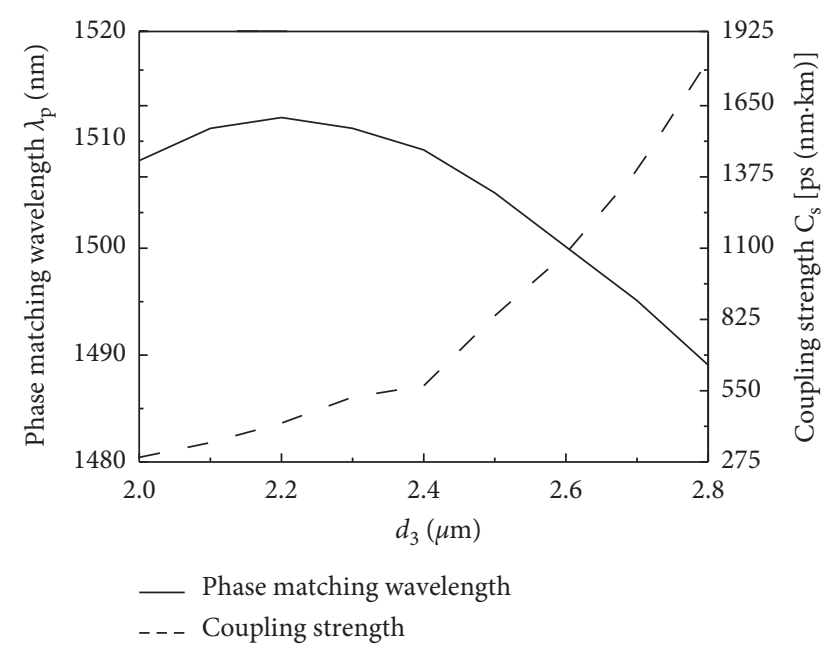

(b)

FIGURE 6: Variation of the effective refractive index of the fundamental supermodel with wavelength when $d_{3}$ is changed (a) and $\lambda p$ and the coupling strength with $d_{3}(\mathrm{~b})$.

time, we can see that the growth amplitude of the fundamental super mode's equivalent refractive index increases with the decrease of $d_{3}$ at short wavelength and long wavelength. So, the change of $d_{3}$ has an influence on the change of the refractive index of inner core and outer core, and the changes of each other are different. Finally, the variation of $\lambda_{\mathrm{p}}$ and coupling strength of the fundamental supermodel is modulated by the refractive index change of the inner core and outer core. The variation of $\lambda_{p}$ and coupling strength with $d_{3}$ is expressed in Figure 6(b). It can be seen in Figure $6(\mathrm{~b})$ that $\lambda_{\mathrm{p}}$ is red shifted first and then blue shifted, but the coupling strength of the fundamental supermodel has been increased, which further shows that this result is adjusted by the refractive index change of the inner core and outer core.

Variation of the effective refractive index of the fundamental supermodel with wavelength when $\Lambda$ is changed is discussed in Figure 7 (a). The effective refractive index of the fundamental supermodel at the short wavelength and the long wavelength increases with the increase of $\Lambda$, but the increase at the short wavelength is less than that at the long wavelength. Meanwhile, the growth amplitude of the equivalent refractive index of the fundamental supermodel at the short wavelength and the long wavelength decreases with the increase of $\Lambda$, respectively. Changing $\Lambda$ is equal to changing the diameter of the cladding and core area, further changing the equivalent refractive index distribution in the cladding and core areas. The changing of equivalent refractive index of cladding also has an influence on the equivalent refractive index distribution in the core areas. The final variation of $\lambda_{\mathrm{p}}$ and coupling strength of the fundamental supermodel is modulated by the variation of the equivalent refractive index of the cladding and core area. The variation of $\lambda_{\mathrm{p}}$ and coupling strength with $\Lambda$ is shown in
Figure $7(b) . \lambda_{p}$ is red shifted and the coupling strength of the fundamental supermodel gradually weakened with the increase of $\Lambda$. Because of the limitation of the structure of the fiber, $\Lambda$ has a small change, so $\lambda_{\mathrm{p}}$ and the coupling strength of the fundamental supermodel produce a single trend under the combined action of the equivalent refractive index of core area and cladding.

The fabrication difficulty of the photonic crystal fiber increases with the increase of the number of layers; we study the number of layers $N=1-3$. Variation of the effective refractive index of the fundamental supermodel with wavelength when $N$ is changed is shown in Figure 8(a). When the number of layers $N$ varies from 1 to 3 , the effective refractive index of the inner core basically remains unchanged, while the refractive index of the outer core increases gradually. But from 2 to 3 layers, the increase of the effective refractive index decreases obviously. The variation of $\lambda_{\mathrm{p}}$ and coupling strength with $N$ is shown in Figure 8(b). $\lambda_{\mathrm{p}}$ is red shifted and the coupling strength becomes larger with the increase of the number of layers $N$, while $\lambda_{\mathrm{p}}$ is less red shifted and the coupling strength has a great change with $N$ changed from 2 to 3 . Therefore, considering the fabrication difficulty of the photonic crystal fiber and the change of the coupling strength with $N$, the number of layers $N=3$ is set in the future research.

3.4. The Result Optimization of the All-Solid DCC-DCPCF. According to the above study of the influence of the fiber structure parameters on $\lambda_{\mathrm{p}}$ and the mode coupling strength, an all-solid DCC-DCPCF is designed for $n_{1}=1.480, n_{2}=1.445, n_{3}=1.430, d_{1}=1.30 \mu \mathrm{m}, d_{2}=0.51 \mu \mathrm{m}$, $d_{3}=3.40 \mu \mathrm{m}, \Lambda=3.50 \mu \mathrm{m}, N=3$, and $N_{\mathrm{o}}=3$. The variation 


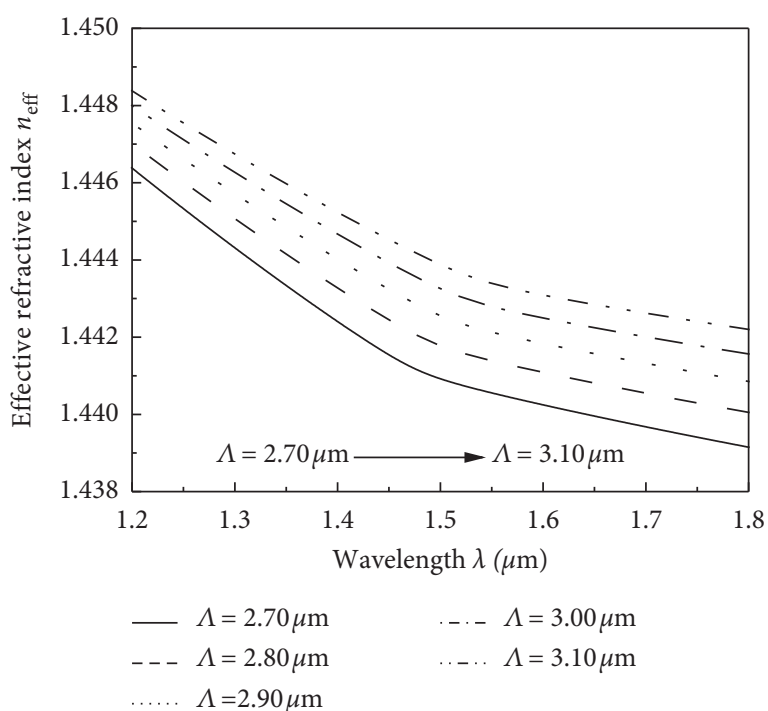

(a)

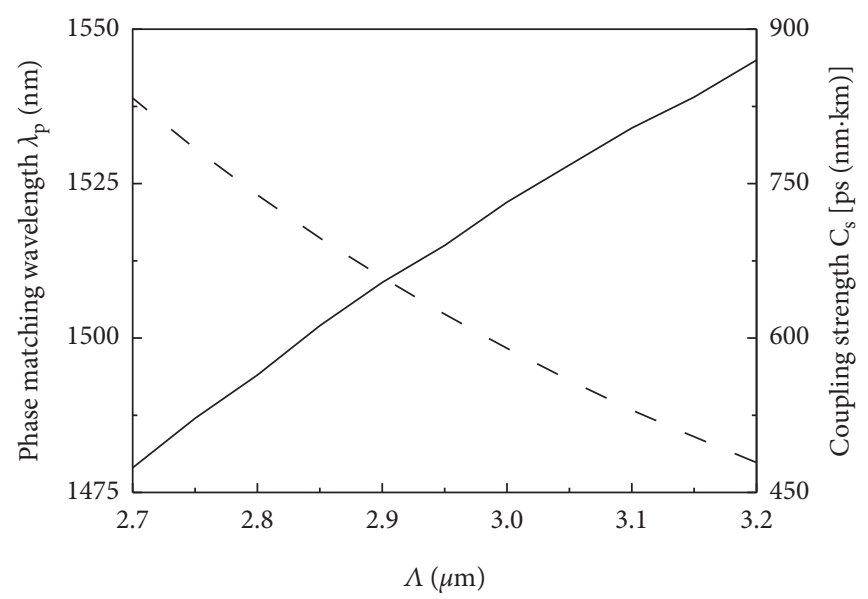

Phase matching wavelength

_. - Coupling strength

FIGURE 7: Variation of the effective refractive index of the fundamental supermodel with wavelength when $\Lambda$ is changed (a) and $\lambda p$ and the coupling strength with $\Lambda$ (b).

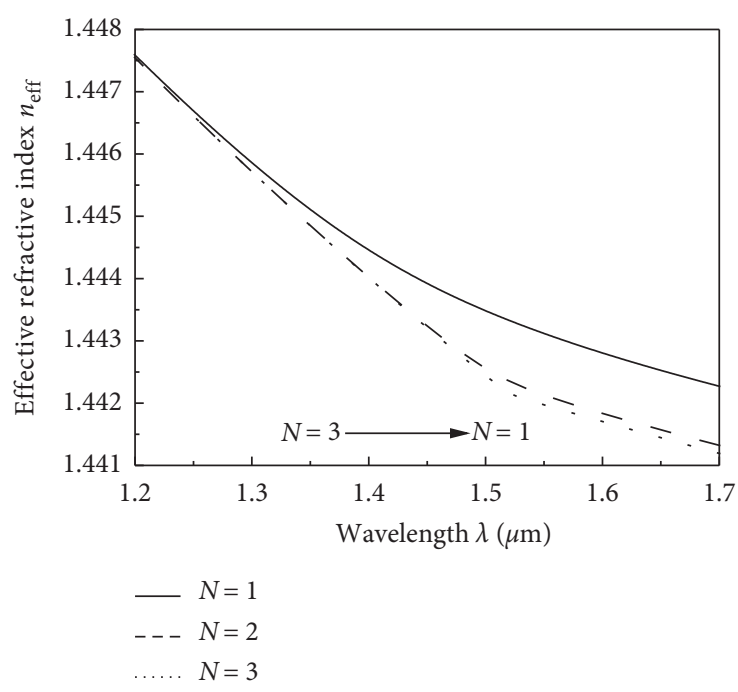

(a)

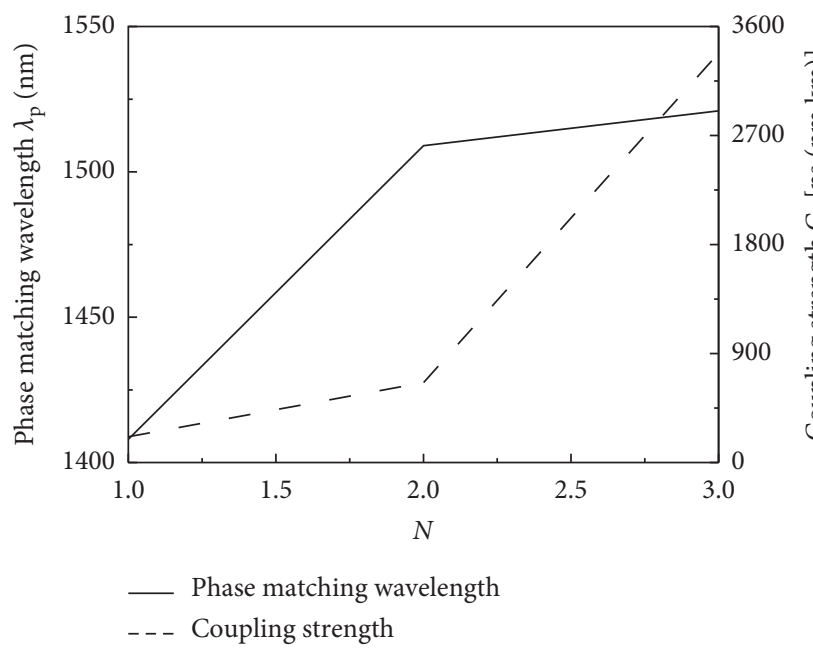

(b)

Figure 8: Variation of the effective refractive index of the fundamental supermodel with wavelength when $N$ is changed (a) and $\lambda p$ and the coupling strength with $N$ (b).

of the dispersion with wavelength is shown in Figure 9. $\lambda_{\mathrm{p}}$ of the designed fiber locates at $1550 \mathrm{~nm}$, and the negative dispersion value reached $-8465 \mathrm{ps} /(\mathrm{nm} \cdot \mathrm{km})$. At $1550 \mathrm{~nm}$, $A_{\text {eff }}$ is $12.8 \mu \mathrm{m}^{2}$ and $L_{\text {splice }}$ is $1.89 \mathrm{~dB}$. The confinement loss and bending loss of the fiber can be controlled by increasing the number of cladding layers. The confinement loss and bending loss with $2 \mathrm{~cm}$ bending radius are less than $1 \times 10^{-3} \mathrm{~dB} / \mathrm{km}$ and $1 \times 10^{-2} \mathrm{~dB} / \mathrm{km}$ at $1550 \mathrm{~nm}$, respectively, when $N_{\mathrm{o}}=6$. The field is mostly confined to the inner core for the optimized DCC-DCPCF. The proposed all-solid DCC-DCPCF not only has large negative dispersion at $1550 \mathrm{~nm}$ and higher compensation ratio, but also has the advantages of low confinement loss and easy fabrication. 


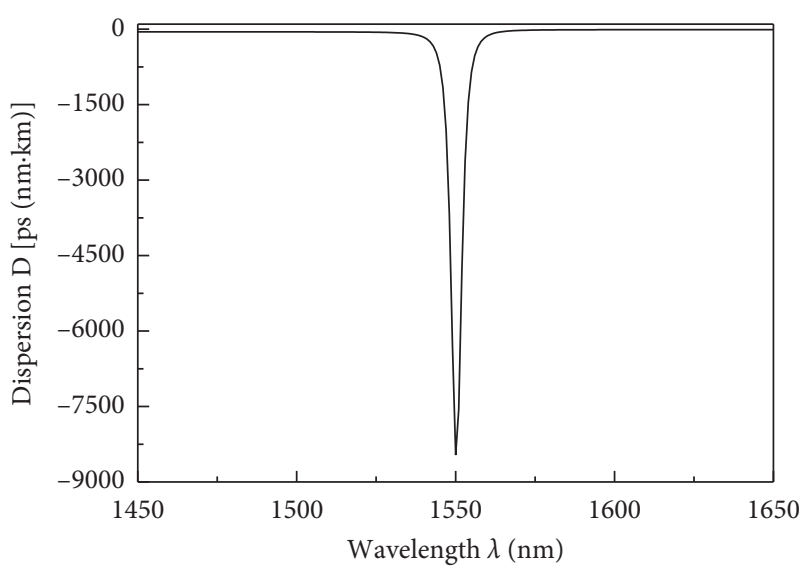

FIGURE 9: Variation of the dispersion with wavelength.

\section{Conclusions}

In summary, an all-solid DCC-DCPCF with large negative dispersion at $1550 \mathrm{~nm}$ has been presented. The influence of the different structure parameters on the mode coupling characteristics is analyzed. The results demonstrate that the proposed all-solid DCC-DCPCF has a large negative dispersion value and low confinement loss. By optimizing the fiber structure parameters, the dispersion value at $1550 \mathrm{~nm}$ of the proposed all-solid DCC-DCPCF researches $-8465 \mathrm{ps} /$ $(\mathrm{nm} \cdot \mathrm{km})$, and the compensation ratio of the proposed fiber to SSMF is 500. The proposed all-solid DCC-DCPCF has important guiding significance for the industrialization of dispersion compensation.

\section{Data Availability}

The data used to support the findings of this study are available from the corresponding author upon request.

\section{Conflicts of Interest}

The authors declare that they have no conflicts of interest regarding the publication of this paper.

\section{Acknowledgments}

This work was supported by the National Natural Science Foundation of China (nos. 61405173 and 61405172) and the Hebei Provincial Natural Science Foundation of China (no. F2018203346).

\section{References}

[1] G. Prabhakar, A. Peer, V. Rastogi, and A. Kumar, "Largeeffective-area dispersion-compensating fiber design based on dual-core microstructure," Applied Optics, vol. 52, no. 19, pp. 4505-4509, 2013.

[2] P. Morin, S. Boivinet, J. P. Yehoussi et al., "Giant pulse at $2 \mu \mathrm{m}$ in polarization maintaining silica step-index fiber," in Proceedings of the Conference on Lasers and Electro-Optics Europe and European Quantum Electronics Conference (CLEO/ Europe-EQEC), Munich, German, June 2019.
[3] T. A. Birks, D. Mogilevtsev, J. C. Knight et al., "Dispersion compensation using single-material fibers," IEEE Photonics Technology Letters, vol. 11, pp. 674-677, 1997.

[4] M. Aliramezani and S. M. Nejad, "Numerical analysis and optimization of a dual-concentric-core photonic crystal fiber for broadband dispersion compensation," Optics \& Laser Technology, vol. 42, no. 8, pp. 1209-1217, 2010.

[5] J. Yuan, X. Sang, C. Yu et al., "Large negative dispersion in dual-concentric-core photonic crystal fiber with hybrid cladding structure based on complete leaky mode coupling," Optics Communications, vol. 284, no. 24, pp. 5847-5852, 2011.

[6] S. K. Varshney, T. Fujisawa, K. Saitoh, and M. Koshiba, "Design and analysis of a broadband dispersion compensating photonic crystal fiber Raman amplifier operating in S-band," Optics Express, vol. 14, no. 8, pp. 3528-3540, 2006.

[7] A. Bala, K. R. Chowdhury, M. B. Mia, and M. Faisal, "Highly birefringent, highly negative dispersion compensating photonic crystal fiber," Applied Optics, vol. 56, no. 25, pp. 7256-7261, 2017.

[8] J.-M. Hsu and G.-S. Ye, "Dispersion ultrastrong compensating fiber based on a liquid-filled hybrid structure of dual-concentric core and depressed-clad photonic crystal fiber," Journal of the Optical Society of America B, Optical Society of America B, vol. 29, no. 8, pp. 2021-2028, 2012.

[9] J.-M. Hsu, W.-H. Zheng, C.-L. Lee, and J.-S. Horng, “Theoretical investigation of a dispersion compensating photonic crystal fiber with ultra-high dispersion coefficient and extremely low confinement loss," Photonics and Nanostructures - Fundamentals and Applications, vol. 16, pp. 1-8, 2015.

[10] P. S. Maji and P. Roy Chaudhuri, "Design of ultra large negative dispersion PCF with selectively tunable liquid infiltration for dispersion compensation," Optics Communications, vol. 325, pp. 134-143, 2014.

[11] L. H. Han, L. M. Liu, Z. Y. Yu et al., "Dispersion compensation properties of dual-concentric core photonic crystal fibers," Chinses Optics Letters, vol. 12, pp. 0106031-0106034, 2014.

[12] H. Xu, J. Wu, K. Xu, Y. Dai, and J. Lin, "Highly nonlinear allsolid photonic crystal fibers with low dispersion slope," Applied Optics, vol. 51, no. 8, pp. 1021-1027, 2012.

[13] H. Xu, X. Wang, X. Huang, C. Zhou, H. Zhu, and X. Cai, “Allsolid photonic crystal fiber for dispersion compensation over," IEEE Photonics Technology Letters, vol. 30, no. 17, pp. 1499-1502, 2018.

[14] K. Mariusz, K. Katarzyna, P. Tomasz et al., "Impact of steepness of pump temporal pulse profile on spectral flatness and correlation of supercontinuum in all-solid photonic crystal fibers with flattened normal dispersion," Journal of Optics, vol. 16, pp. 0852021-0852028, 2014.

[15] C. Wang, Y. Zhang, Z. Wu et al., "Design of all-solid dualconcentric-core microstructure fiber for ultra-broadband dispersion compensation," Applied Sciences, vol. 9, pp. 33661-33669, 2019.

[16] A. Kumar, T. S. Saini, K. D. Naik et al., "Large-mode-area single-polarization single-mode photonic crystal fiber: design and analysis," Applied Optics, vol. 55, pp. 4995-5000, 2016.

[17] M. De, R. K. Gangwar, and V. K. Singh, "Designing of highly birefringence, dispersion shifted decagonal photonic crystal fiber with low confinement loss," Photonics and Nanostructures, vol. 26, pp. 15-23, 2017.

[18] M. Seifouri, M. Dekamin, and S. Olyaee, "A new circular chalcogenide/silica hybrid microstructured optical fiber with high negative dispersion for the purpose of dispersion compensation," Optik, vol. 126, pp. 3093-3098, 2015. 
[19] W. Wang, Y. W. Qu, C. L. Zhang et al., "Novel design of broadband dispersion compensating photonic crystal fiber with all solid structure and low index difference," Optik, vol. 156, pp. 279-288, 2018.

[20] X. Y. Li, P. Liu, Z. L. Xu et al., "Design of a pentagonal photonic crystal fiber with high birefringence and large flattened negative dispersion," Applied Optics, vol. 54, pp. 7350-7357, 2015.

[21] Y. Tsuchida, K. Saitoh, and M. Koshiba, "Design and characterization of single-mode holey fibers with low bending losses," Optics Express, vol. 13, pp. 4770-4779, 2005.

[22] X. F. Zhou, Z. L. Chen, H. H. Chen et al., "Fusion splicing small-core photonic crystal fibers and single-mode fibers by controlled air hole collapse," Optics Communications, vol. 285, pp. 5283-5286, 2012.

[23] M. Damian, S. Tomasz, and B. Ryszard, "Dispersion management in hybrid optical fibers," Journal of Lightwave Technology, vol. 38, pp. 1427-1434, 2020.

[24] F. Gerome, J.-L. Auguste, and J.-M. Blondy, "Design of dispersion-compensating fibers based on a dual-concentric-core photonic crystal fiber," Optics Letters, vol. 29, no. 23, pp. 2725-2727, 2004. 